Copyright 2001 American Association of Physics Teachers (AIP Publishing). This article may be downloaded for personal use only. Any other use requires prior permission of the author and AIP Publishing.

The following article appeared as: Penner, A.R. (2001). The physics of golf: The convex face of a driver. American Journal of Physics, 69(10), 1073-1081, and may be found at http://dx.doi.org/10.1119/1.1380380 


\title{
The physics of golf: The convex face of a driver
}

\author{
A. Raymond Penner \\ Physics Department, Malaspina University-College, Nanaimo, British Columbia, Canada
}

(Received 30 November 2000; accepted 28 March 2001)

\begin{abstract}
The impact of the clubhead of a driver with a golf ball is modeled. The effect of the convex clubface of a driver on the flight of the golf ball is considered and the dependence of the optimum curvature
\end{abstract} of the clubface on the volume, mass, and impact speed of the clubhead is determined. (C) 2001

American Association of Physics Teachers.

[DOI: $10.1119 / 1.1380380]$

\section{INTRODUCTION}

Golf is not only an extremely popular game but is also a storehouse of physics problems. This paper will concern itself with one such problem, and that is why the clubface of a driver, the golf club used to drive the golf ball the maximum distance, has a convex curvature. This is a perplexing problem for it would be thought that a convex face would be the last thing that would be wanted as any off-center impact would be expected to fly even further off target. The goal of this paper is therefore to determine what effect the curvature of a clubface has on the subsequent flight and run of the golf ball. A mathematical model of the general eccentric impact between the clubhead of a driver and a golf ball will be presented which will provide a quantitative explanation of the curvature of the clubface. In addition, this model will also allow for the determination of the optimum radius of curvature for the clubface for a specific impact point, and its dependence on clubhead parameters.

Although Cochran and Stobbs ${ }^{1}$ do consider the convex nature of a driver's clubface, this goes only so far as a descriptive explanation. This is also true for the description as given by Maltby ${ }^{2}$ in his golf club design book, although in this case some empirical results are also provided. Mathematical models of the impact between a clubhead and a golf ball provided by Daish, ${ }^{3}$ Jorgensen, ${ }^{4}$ and Penner ${ }^{5}$ are also limited in that only central impacts are considered, in which case the curvature of the clubface will have no effect. Only by considering eccentric impacts between a golf ball and a clubhead can the effect of the curvature of the clubface be determined.

\section{SPIN AND ITS EFFECT ON THE FLIGHT OF A GOLF BALL}

To understand why the face of a driver is convex, it is first necessary to understand the direction of the spin that is imparted to a golf ball during impact. The direction of the spin will be determined by the direction of the velocity component of the clubface at the impact point which is tangent to the clubface. In general, there are two cases where the clubface will have a velocity component tangent to its surface at the impact point. The first is the case where the normal to the clubface at the impact point is not parallel to the clubhead's velocity, $\mathbf{v}_{\mathbf{c}}$. An example of this is given in Fig. 1(a) where the side view of a driver is shown. Due to the loft of the clubhead, the velocity, $\mathbf{v}_{\mathbf{a}}$, of the impact point, a, will have a component, $v_{a p}$, pointing down, parallel to the clubface, and a component, $v_{a n}$, normal to the clubface. The direction of $v_{a p}$ will determine the direction of the resulting frictional force acting on the ball's surface during impact and will therefore determine the direction of the spin. In the case shown in Fig. 1(a) the result will be that the ball will acquire backspin. Another example is given in Fig. 1(b), which shows the overhead view of a driver with a convex face. In the case of an off-center impact with the golf ball, the clubface will have a tangential velocity component, $v_{a t}$, at the impact point pointing toward the clubface's center. This will impart a sidespin to the golf ball in the clockwise direction as shown in the figure. This effect where sidespin is imparted to a golf ball due to the normal to the clubface at the impact point being at an angle with respect to the velocity of the clubhead will be referred to in this paper as the "angle effect."

The second way that a golf ball is given spin by the impact with the golf club arises from the rotation of the clubhead. Figure 1(c) shows the overhead view of a driver with a flat face. In this case the normal to the clubface is parallel to the clubhead's velocity, so no angle effect will be present. However, in the case of an off-center impact, an angular velocity, $\omega_{\mathrm{c}}$, will be imparted to the clubhead. This will result in the velocity of the impact point of the clubface, $\mathbf{v}_{\mathbf{a}}$, being

$$
\mathbf{v}_{\mathbf{a}}=\mathbf{v}_{\mathbf{c}}+\omega_{\mathbf{c}} \times \mathbf{r}_{\mathbf{c a}}
$$

where $\mathbf{r}_{\mathbf{c a}}$ is the position of the impact point relative to the center of mass of the clubhead. As shown in the figure, this will result in the impact point having a velocity component, $v_{a t}$, tangent to its surface pointing away from the center of the face. This will impart a sidespin to the golf ball in the counterclockwise direction for the case shown in Fig. 1(c). This effect is what is normally referred to in golf as the "gear effect." As seen in Figs. 1(b) and 1(c), in terms of the orientation of the sidespin given to the golf ball, the angle effect and the gear effect generally oppose each other. Which effect is dominant will depend on the position of the impact point, on the curvature of the face, on the mass and moments of inertia of the clubhead, and on the clubhead's speed at impact. For example, if the curvature of the clubface is relatively large, the angle effect will typically be dominant and the golf ball will end up with the sidespin shown in Fig. 1(b). If the curvature of the clubface is relatively small, the gear effect will typically dominate and the golf ball will end up with the sidespin shown in Fig. 1(c).

The orientation of the spin which is imparted to the golf ball will in turn determine the direction of the Magnus force which acts on the spinning golf ball as it moves through the air. The direction of the Magnus force is, in general, given by $\hat{\omega}_{\mathbf{b}} \times \hat{\mathbf{v}}_{\mathbf{b}}$, where $\hat{\omega}_{\mathbf{b}}$ is the unit vector in the direction of the angular velocity and $\hat{\mathbf{v}}_{\mathbf{b}}$ is the unit vector in the direction of 


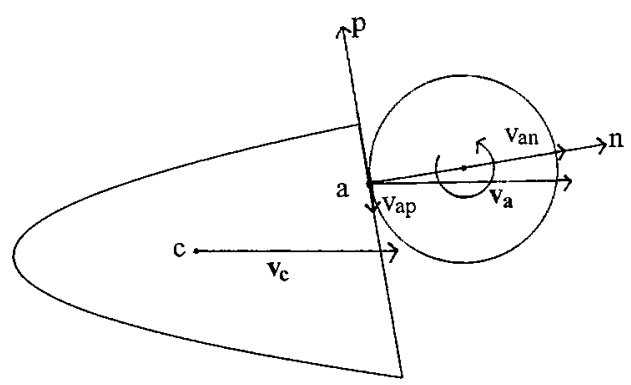

a)

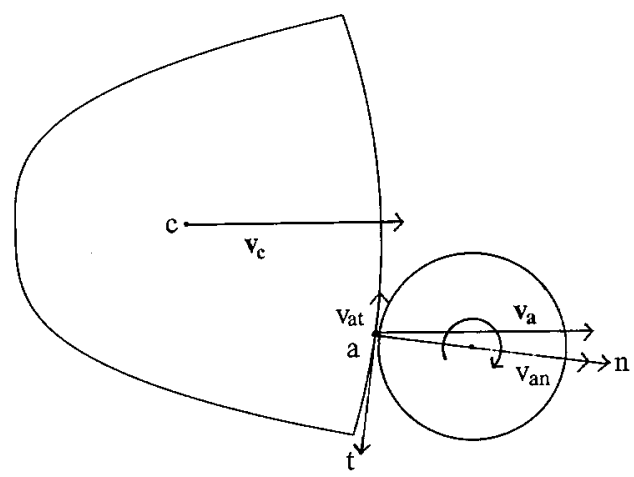

b)

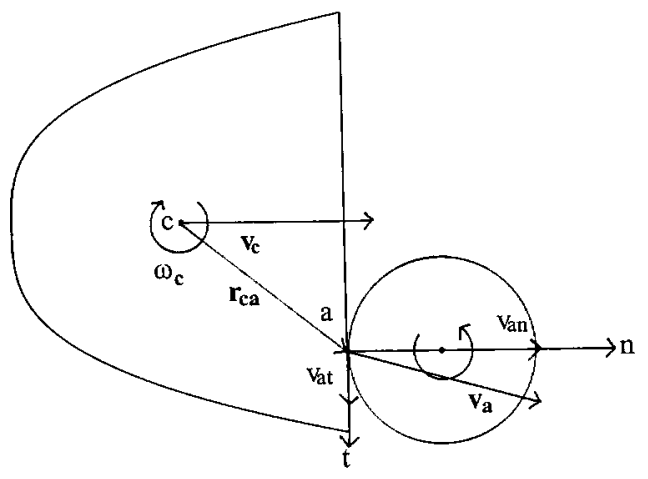

c)

Fig. 1. Examples where the clubface has a velocity component tangent to its surface at the impact point.

the linear velocity. As an example, Fig. 2 shows the sideview of a golf ball struck with a driver and thereby being given backspin. As shown in the figure, the result is that the Magnus force, $\mathbf{F}_{\mathbf{M}}$, will have a positive vertical component. A golf ball will travel significantly further through the air because of this effect. Figure 3 shows the overhead views of off-center hits by a driver. In these cases the ball is given sidespin, which will result in the Magnus force having a component in the horizontal direction, perpendicular to the balls velocity. The ball will therefore curve sideways in flight. Figure 3(a) shows the case where the face of the driver is flat and only the gear effect is present. For an off-center impact the Magnus force will cause the golf ball to curve across the center of the fairway. Figure 3(b) shows the case where the face is given only a slight curvature so that the gear effect is dominant and the ball will therefore start off in the direction perpendicular to the clubface but will curve

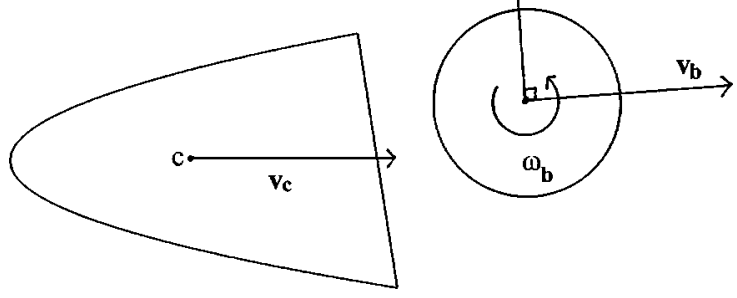

Fig. 2. The direction of the Magnus force, $\mathbf{F}_{\mathbf{M}}$, in the case where the golf ball is imparted back spin.

back, due to the Magnus force, toward the center of the fairway. In the case shown in Fig. 3(b), where the curvature is just right the net effect will be that the golf ball will end up landing in the middle of the fairway. Figure 3(c) shows the case where the curvature of the clubface is too great, resulting in the angle effect dominating for an off-center impact. The resulting Magnus force will cause the ball which starts out in the direction perpendicular to the clubface to veer even further away from the center of the fairway. It is now clear why the face of a driver is slightly convex. As shown in Fig. 3(b), it will compensate for the effect of the sidespin imparted to a golf ball as a result of the gear effect in the case of off-center impacts. To determine quantitatively the optimum amount of curvature that should be given to the clubface of a driver it will be necessary to have appropriate models for the clubhead, for the impact with the golf ball, and for the subsequent trajectory.

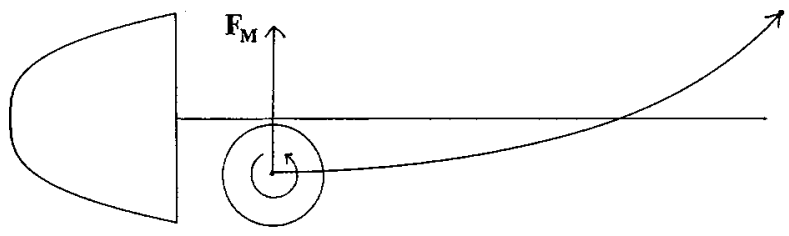

a)

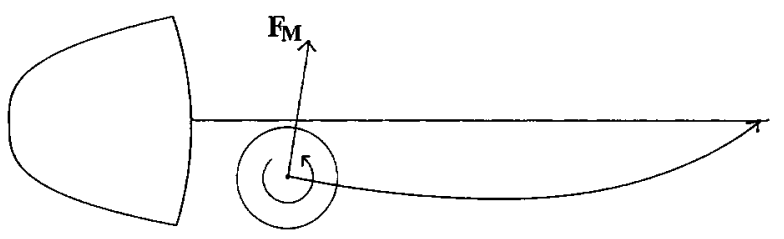

b)

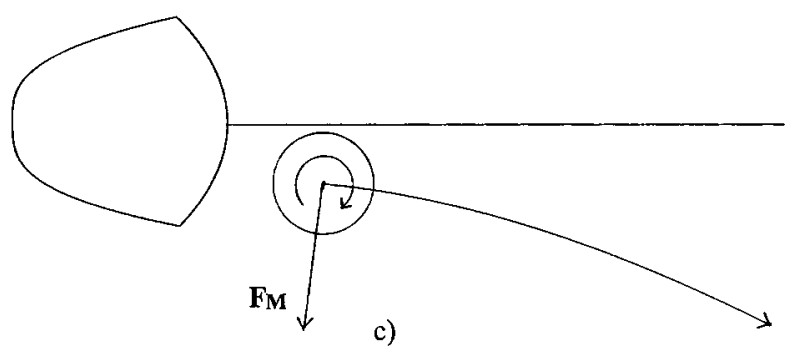

Fig. 3. Examples of the resulting golf ball trajectories due to the Magnus force, $\mathbf{F}_{\mathbf{M}}$. 


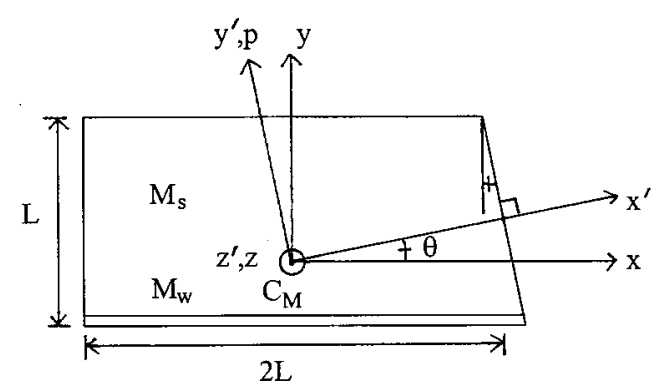

a)

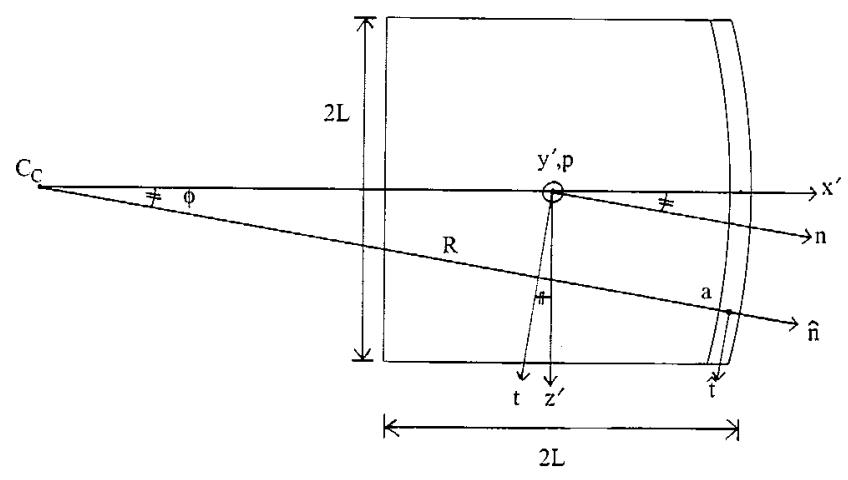

b)

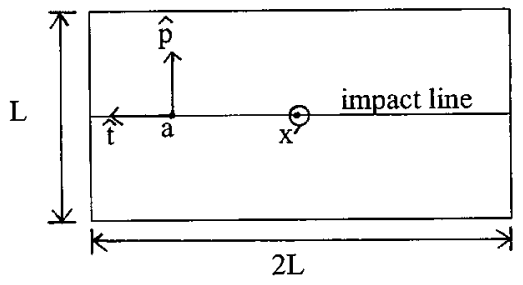

c)

Fig. 4. The geometry of the clubhead model; (a) side view, (b) overhead view, (c) front view.

\section{MODEL OF THE CLUBHEAD}

Modern driver clubheads are hollow and are aerodynamically shaped in order to reduce the air drag during the swing. However, for the purposes of this paper the clubhead can be adequately modeled as a rectangular shell of height $L$, and with a length and width of $2 L$. In a typical driver, weight is added to the sole of the clubhead in order to align the center of mass along the normal to the center of the clubface. This optimizes the velocity imparted to the golf ball for central impacts. In the case of the above clubhead model, this is accomplished by adding a plate of appropriate mass, $M_{w}$, to the sole of the shell, which will be taken to have a mass of $M_{s}$. The geometry of the model is shown in Figs. 4(a)-4(c), with the face of the driver set at a loft of $\theta$, and the radius of curvature of the cylindrically shaped clubface being set at $R$. The center of mass of the clubhead, $C_{M}$, will be at a perpendicular distance of $L / \cos \theta$ from the center of the clubface and will be taken as the origin of the coordinate systems shown in the figures. The velocity of the clubhead will be taken to be along the $x$ axis, while the $x^{\prime}$ axis is perpendicular to the center of the face of the driver. The $n$ axis will be taken to be in a direction normal to the clubface at the point of impact, $a$. The $t$ axis is in a direction tangent to the curved face at the impact point while the $p$ axis is in a direction parallel to the loft of the clubhead. The angle $\phi$ gives the position of the impact point with respect to the center of curvature of the clubface, $C_{c}$. For simplicity, this paper will only consider impacts on the horizontal line, as shown in Fig. 4(c), that run along the center of the face.

The masses, $M_{s}$ and $M_{w}$, required to place the center of mass along the normal to the center of the clubface, the $x^{\prime}$ axis, are determined to be

$$
\begin{aligned}
& M_{w}=(2 \tan \theta) M, \text { and } \\
& M_{s}=M-M_{w},
\end{aligned}
$$

where $M$ is the total mass of the club. As an example, for a typical driver clubhead of volume $250 \mathrm{cc}$, mass $200 \mathrm{~g}$, and loft of $10.5^{\circ}$ the values for $M_{w}, M_{s}$, and $L$ are $74.1 \mathrm{~g}, 125.9$ $\mathrm{g}$, and $3.97 \mathrm{~cm}$, respectively. The moments of inertia about the $x, y$, and $z$ axes for the given clubhead model are found to be

$$
\begin{aligned}
& I_{\mathrm{c} x}=\left(2 / 3-1 / 6 \tan \theta-\tan \theta^{2}\right) M L^{2}, \\
& I_{\mathrm{c} z}=I_{\mathrm{c} x}, \quad \text { and } \\
& I_{\mathrm{c} y}=(1-2 / 3 \tan \theta) M L^{2} .
\end{aligned}
$$

These expressions were determined by treating the clubhead as a rectangular shell with a weighted sole and ignoring the effects of the loft and the curvature of the clubface. The resulting $y$ component of the moment of inertia, $I_{\mathrm{c} y}$, for the driver clubhead values given above, is found from Eq. (3c) to be $2.76 \times 10^{3} \mathrm{~g} \mathrm{~cm}^{2}$. This is only slightly less than the experimental value of $3.1 \times 10^{3} \mathrm{~g} \mathrm{~cm}^{2}$ found about the same axis for a similar clubhead volume. ${ }^{6}$ The moments of inertia about the $n, t$, and $p$ axes for a given impact position, $\phi$, are found, by suitable transformations, to be

$$
\begin{aligned}
& I_{\mathrm{c} n}=I_{\mathrm{c} z} \sin ^{2} \phi+I_{\mathrm{c} x} \cos ^{2} \phi \cos ^{2} \theta+I_{\mathrm{c} y} \cos ^{2} \phi \sin ^{2} \theta, \\
& I_{\mathrm{c} p}=I_{\mathrm{c} x} \sin ^{2} \theta+I_{\mathrm{c} y} \cos ^{2} \theta, \text { and } \\
& I_{\mathrm{c} t}=I_{\mathrm{c} z} \cos ^{2} \phi+I_{\mathrm{c} x} \sin ^{2} \phi \cos ^{2} \theta+I_{\mathrm{c} y} \sin ^{2} \phi \sin ^{2} \theta .
\end{aligned}
$$

The golf ball will be modeled as a uniform solid sphere with the following moments of inertia:

$$
I_{\mathrm{b} n}=I_{\mathrm{b} p}=I_{\mathrm{b} t}=(2 / 5) \mathrm{m} \mathrm{r}^{2},
$$

where $m$ is the mass of the golf ball and $r$ is its radius. The USGA regulations set the minimum diameter of a golf ball at $4.27 \mathrm{~cm}$ (1.68 in.) and the maximum mass at $45.9 \mathrm{~g}(1.62$ $\mathrm{oz}$ ). Using these values for the mass and size of a golf ball results in a value of $83.7 \mathrm{~g} \mathrm{~cm}^{2}$ for the moments of inertia which are given by Eq. (5). This is slightly greater than the experimental values for the moments of inertia of similarly sized golf balls which ranged from 72.8 to $81.3 \mathrm{~g} \mathrm{~cm}^{2}$.

\section{MODEL OF THE IMPACT}

The general collision between a clubhead and a golf ball is an example of a three-dimensional eccentric impact. The standard method of analysis ${ }^{8,9}$ for such a problem is to consider the linear and angular impulse-momentum laws and the constraints on the velocities. Although this method cannot describe the transient stresses and deformations produced, it will allow for the determination of the initial and final veloc- 
ity states of the colliding objects and the applied impulse. In order to simplify the analysis, several assumptions or approximations will be required. The first assumption is that the clubhead behaves as a free body during impact, in that the effect of the shaft can be ignored. This is a reasonable approximation as the impact force will be much larger than the force the shaft exerts on the clubhead during the collision. This was demonstrated by Cochran and Stobbs, ${ }^{1}$ where tests were made with a clubhead hinged on a golf club shaft. Flash photographs and the resulting flight of the ball showed that this unique shaft had little effect on the impact. A second approximation that will be made is that the impact occurs at a single point, although photographs have shown that a golf ball is significantly flattened against the face of the clubhead. A third approximation is that the impact is instantaneous and therefore there is no change in the orientation or position of the clubhead during the collision. A fourth approximation is that the golf ball is in a state of pure rolling when it leaves the clubface. This follows from the experimental results of Cochran and Stobbs ${ }^{1}$ and of Chou et al., ${ }^{10}$ who showed that for clubface lofts below $40^{\circ}$ the spin of the launched golf ball was approximately independent of the smoothness of the clubface. As stated, these approximations will simplify the analysis and will not affect any of the general conclusions.

The collision between a clubhead and a golf ball will be analyzed with respect to the $n, p$, and $t$ axes shown in Fig. 4. The impulse acting on the clubhead will be taken to be

$$
\mathbf{P}=P_{n} \hat{\mathbf{n}}+P_{p} \hat{\mathbf{p}}+P_{t} \hat{\mathbf{t}} .
$$

The change in the linear momentum of the clubhead will then be given by

$$
M\left(\mathbf{v}_{\mathbf{c f}}-\mathbf{v}_{\mathbf{c i}}\right)=\mathbf{P},
$$

where $\mathbf{v}_{\mathbf{c i}}$ is the initial clubhead velocity and $\mathbf{v}_{\mathbf{c f}}$ is the final clubhead velocity. The initial clubhead velocity is taken to be along the $x$ axis and will therefore be given by

$$
\begin{aligned}
\mathbf{v}_{\mathbf{c i}} & =v_{\mathrm{ci}} \hat{\mathbf{x}} \\
& =\left(v_{\mathrm{ci}} \cos \theta \cos \phi\right) \hat{\mathbf{n}}-\left(v_{\mathrm{ci}} \sin \theta\right) \hat{\mathbf{p}}-\left(v_{\mathrm{ci}} \cos \theta \sin \phi\right) \hat{\mathbf{t}} .
\end{aligned}
$$

The final linear momentum of the golf ball will in turn be given by

$$
m_{\mathrm{b}} \mathbf{v}_{\mathbf{b f}}=-\mathbf{P},
$$

where $\mathbf{v}_{\mathbf{b f}}$ is the velocity of the golf ball after impact.

The final angular momentum of the clubhead, $\mathbf{H}_{\mathbf{c f}}$, about its center of mass will be

$$
\mathbf{H}_{\mathbf{c f}}=\mathbf{r}_{\mathrm{ca}} \times \mathbf{P},
$$

where $\mathbf{r}_{\mathbf{c a}}$, the position vector from the clubhead's center of mass to the impact point, is given by

$$
\begin{aligned}
\mathbf{r}_{\mathbf{c a}}= & R \hat{\mathbf{n}}-(R-L / \cos \theta) \hat{\mathbf{x}} \\
= & (R(1-\cos \phi)+L \cos \phi / \cos \theta) \hat{\mathbf{n}} \\
& +(R-L / \cos \theta) \sin \phi \hat{\mathbf{t}}
\end{aligned}
$$

$$
=r_{\mathrm{c} a n} \hat{\mathbf{n}}+r_{\mathrm{c} a t} \hat{\mathbf{t}} \text {. }
$$

For moments of inertia of $I_{\mathrm{c} n}, I_{\mathrm{c} p}$, and $I_{\mathrm{c} t}$ about the $n, p$, and $t$ axes, respectively,

$$
\mathbf{H}_{\mathrm{cf}}=I_{\mathrm{c} n} \omega_{\mathrm{cf} n} \hat{\mathbf{n}}+I_{\mathrm{c} p} \omega_{\mathrm{cf} p} \hat{\mathbf{p}}+I_{\mathrm{cf}} \omega_{\mathrm{cft} t} \hat{\mathbf{t}}
$$

where $\omega_{\mathrm{cf} n}, \omega_{\mathrm{cf} p}$, and $\omega_{\mathrm{cf} t}$ represent the final angular velocities of the clubhead about the specified axes. The final angular momentum of the golf ball, $\mathbf{H}_{\mathbf{b f}}$, about its center of mass, will be

$$
\mathbf{H}_{\mathbf{b f}}=\mathbf{r}_{\mathrm{ba}} \times(-\mathbf{P}),
$$

where $\mathbf{r}_{\mathbf{b a}}$, the position vector from the golf ball's center of mass to the impact point, is given by

$$
\mathbf{r}_{\mathbf{b a}}=-r \hat{\mathbf{n}} .
$$

For moments of inertia of $I_{\mathrm{b} n}, I_{\mathrm{b} p}$, and $I_{\mathrm{b} t}$ about the $n, p$, and $t$ axes, respectively,

$$
\mathbf{H}_{\mathrm{bf}}=I_{\mathrm{b} n} \omega_{\mathrm{bf} n} \hat{\mathbf{n}}+I_{\mathrm{b} p} \omega_{\mathrm{bf} p} \hat{\mathbf{p}}+I_{\mathrm{b} t} \omega_{\mathrm{b} t} \hat{\mathbf{t}},
$$

where $\omega_{\mathrm{bf} n}, \omega_{\mathrm{bf} p}$, and $\omega_{\mathrm{bft}}$ represent the final angular velocities of the golf ball about the specified axes.

There are two constraints on the final velocities of the clubhead and the golf ball. The coefficient of restitution, $e$, can be defined as the ratio of the final to the initial relative velocity along the line of impact and is related to the loss of mechanical energy during the collision. In terms of the velocities of the clubhead and golf ball, this constraint leads to

$$
\left(\mathbf{v}_{\mathbf{b f}}+\omega_{\mathbf{b f}} \times \mathbf{r}_{\mathbf{b a}}\right) \cdot \hat{\mathbf{n}}-\left(\mathbf{v}_{\mathbf{c f}}+\omega_{\mathbf{c f}} \times \mathbf{r}_{\mathbf{c a}}\right) \cdot \hat{\mathbf{n}}=e \mathbf{v}_{\mathbf{c i}} \cdot \hat{\mathbf{n}} .
$$

Measurements by Lieberman and Johnson ${ }^{11}$ of golf balls fired into a stationary steel plate have given values for $e$ decreasing from approximately 0.76 for impact velocities of $37 \mathrm{~m} / \mathrm{s}$ to values of approximately 0.72 for impact velocities of $50 \mathrm{~m} / \mathrm{s}$. Applying a linear fit to these results gives the following dependence of $e$ on impact speed and loft:

$$
e=0.86-0.0029 v_{\mathrm{ci}} \cos \theta .
$$

The approximation that the ball is in a state of rolling when it leaves the clubface is equivalent to setting the final relative tangential velocity at the impact point equal to zero. Applying this constraint to both the $t$ and $p$ axes results in

$$
\left(\mathbf{v}_{\mathbf{c f}}+\omega_{\mathbf{c f}} \times \mathbf{r}_{\mathbf{c a}}\right) \cdot \hat{\mathbf{t}}-\left(\mathbf{v}_{\mathbf{b f}}+\omega_{\mathbf{b f}} \times \mathbf{r}_{\mathbf{b a}}\right) \cdot \hat{\mathbf{t}}=0,
$$
and

$$
\left(\mathbf{v}_{\mathbf{c f}}+\omega_{\mathbf{c f}} \times \mathbf{r}_{\mathbf{c a}}\right) \cdot \hat{\mathbf{p}}-\left(\mathbf{v}_{\mathbf{b f}}+\omega_{\mathbf{b f}} \times \mathbf{r}_{\mathbf{b a}}\right) \cdot \hat{\mathbf{p}}=0 .
$$

The six linear equations for the linear momentum, Eqs. (7) and (9), six linear equations for the angular momentum, Eqs. (10) and (13), and the three linear equations of constraint, Eqs. (16) and (18), can be solved for the 15 components of $\mathbf{v}_{\mathbf{c f}}, \mathbf{v}_{\mathbf{b f}}, \omega_{\mathbf{c f}}, \omega_{\mathbf{b f}}$, and $\mathbf{P}$. The solution for the final linear velocity of the golf ball is as follows:

$$
\mathbf{v}_{\mathbf{b f}}=v_{\mathrm{bf} n} \hat{\mathbf{n}}+v_{\mathrm{bf} p} \hat{\mathbf{p}}+v_{\mathrm{bft}} \hat{\mathbf{t}}
$$

with

$$
v_{\mathrm{bf} n}=v_{\mathrm{ci}} \cos \theta \frac{(1+e) \gamma I_{\mathrm{c} p} \cos \phi+(1+e) m r_{\mathrm{c} a n}^{2} \cos \phi-m r_{\mathrm{c} a n} r_{\mathrm{c} a t} \sin \phi}{\beta \gamma I_{\mathrm{c} p}+\gamma m r_{\mathrm{c} a t}^{2}+\beta m r_{\mathrm{c} a n}^{2}}
$$




$$
v_{\mathrm{bf} p}=-v_{\mathrm{ci}} \sin \theta / \Delta,
$$

and

$$
\begin{aligned}
v_{\mathrm{bft} t}= & -v_{\mathrm{bf} n} \frac{\beta r_{\mathrm{c} a n}}{\gamma r_{\mathrm{c} a t}} \\
& -v_{\mathrm{ci}} \cos \theta \frac{r_{\mathrm{c} a t} \sin \phi-(1+e) r_{\mathrm{c} a n} \cos \phi}{\gamma r_{\mathrm{c} a t}},
\end{aligned}
$$

where

$$
\begin{aligned}
& \beta=(1+m / M), \\
& \gamma=\left(1+m / M+m r^{2} / I_{\mathrm{b} p}\right),
\end{aligned}
$$

and

$$
\Delta=\left(1+m / M+m r^{2} / I_{\mathrm{b} t}+m r_{\mathrm{c} a n}^{2} / I_{\mathrm{c} t}+m r_{\mathrm{c} a t}^{2} / I_{\mathrm{c} n}\right) .
$$

The final angular velocity of the golf ball is given by

$$
\omega_{\mathrm{bf}}=\omega_{\mathrm{bf} n} \hat{\mathbf{n}}+\omega_{\mathrm{bf} p} \hat{\mathbf{p}}+\omega_{\mathrm{bf} t} \hat{\mathbf{t}},
$$

where

$$
\begin{aligned}
& \omega_{\mathrm{bf} n}=0, \\
& \omega_{\mathrm{bf} p}=v_{\mathrm{bf} t} m r / I_{\mathrm{b} p},
\end{aligned}
$$

and

$$
\omega_{\mathrm{bf} t}=-v_{\mathrm{bf} p} m r / I_{\mathrm{b} t} .
$$

The solution for the final linear velocity of the clubhead is given by

$$
\mathbf{v}_{\mathrm{cf}}=v_{\mathrm{cf} n} \hat{\mathbf{n}}+v_{\mathrm{cf} p} \hat{\mathbf{p}}+v_{\mathrm{cf} t} \hat{\mathbf{t}},
$$

where

$$
\begin{aligned}
& v_{\mathrm{cf} n}=v_{\mathrm{ci}} \cos \theta \cos \phi-(m / M) v_{\mathrm{bf} n}, \\
& v_{\mathrm{cf} p}=-v_{\mathrm{ci}} \sin \theta-(m / M) v_{\mathrm{bf} p},
\end{aligned}
$$

and

$$
v_{\mathrm{cf} t}=-v_{\mathrm{ci}} \cos \theta \sin \phi-(m / M) v_{\mathrm{bf} t} .
$$

The final angular velocity of the clubhead is given by

$$
\omega_{\mathrm{cf}}=\omega_{\mathrm{cf} n} \hat{\mathbf{n}}+\omega_{\mathrm{cf} p} \hat{\mathbf{p}}+\omega_{\mathrm{cff}} \hat{\mathbf{t}},
$$

where

$$
\begin{aligned}
& \omega_{\mathrm{cf} n}=v_{\mathrm{bf} p} m r_{\mathrm{c} a t} / I_{\mathrm{c} n}, \\
& \omega_{\mathrm{cf} p}=v_{\mathrm{bft}} m r_{\mathrm{c} a n} / I_{\mathrm{c} p}-v_{\mathrm{bf} n} m r_{\mathrm{c} a t} / I_{\mathrm{c} p},
\end{aligned}
$$

and

$$
\omega_{\mathrm{cft} t}=-v_{\mathrm{bf} p} m r_{\mathrm{c} a n} / I_{\mathrm{c} t} .
$$

The final velocity of the clubface at the impact point can be determined using Eq. (1). Of particular interest is the component of the velocity along the $t$ axis, as it will determine whether the gear effect or angle effect is dominant. This component is given by

$$
\mathbf{v}_{\mathbf{a}} \cdot \hat{\mathbf{t}}=v_{\mathrm{cft}}-\omega_{\mathrm{cf} p} r_{\mathrm{c} a n} .
$$

\section{FLIGHT AND RUN OF A GOLF BALL}

The flight of a golf ball through the air has been considered in several articles presented in this Journal. Bearman and Harvey, ${ }^{12}$ Erlichson, ${ }^{13}$ MacDonald and Hanzely, ${ }^{14}$ along with Penner, ${ }^{5}$ considered the two-dimensional trajectory of golf balls with backspin while McPhee ${ }^{15}$ considered the additional effect of sidespin.

In order to model the three-dimensional trajectories of golf balls the forces due to gravity, air drag, and the Magnus effect need to be determined. The gravitational force, $\mathbf{F}_{\mathbf{G}}$, is given by

$$
\mathbf{F}_{\mathbf{G}}=-m g \hat{\mathbf{y}}
$$

and will be constant throughout the flight. The air drag, $\mathbf{F}_{\mathbf{D}}$, acting on the ball at any point along its path is, in general, given by

$$
\mathbf{F}_{\mathbf{D}}=-1 / 2 \rho\left(\pi r^{2}\right) C_{D} v_{b}^{2} \hat{\mathbf{v}}_{\mathbf{b}},
$$

where $\rho$ is the density of the air, $1.205 \mathrm{~kg} / \mathrm{m}^{3}$ for dry air at $20^{\circ} \mathrm{C}, C_{D}$ is the drag coefficient, $v_{b}$ is the speed of the ball, and $\hat{\mathbf{v}}_{\mathbf{b}}$ is the unit vector in the direction of motion. The initial flight values of $v_{b}$ and $\hat{\mathbf{v}}_{\mathbf{b}}$ are given by

$$
v_{\mathrm{b} 0}=v_{\mathrm{bf}}=\left(v_{\mathrm{bf} n}^{2}+v_{\mathrm{bf} t}^{2}+v_{\mathrm{bf} p}^{2}\right)^{1 / 2}
$$

and

$$
\begin{aligned}
\hat{\mathbf{v}}_{\mathrm{b} 0}= & \left(v_{\mathrm{bf} n} \hat{\mathbf{n}}+v_{\mathrm{bf} t} \hat{\mathbf{t}}+v_{\mathrm{bf} p} \hat{\mathbf{p}}\right) / v_{\mathrm{b} 0} \\
= & \left(\left(v_{\mathrm{bf} n} \cos \phi \cos \theta-v_{\mathrm{bf} t} \sin \phi \cos \theta-v_{\mathrm{bf} p} \sin \theta\right) \hat{\mathbf{x}}\right. \\
& +\left(v_{\mathrm{bf} n} \cos \phi \sin \theta-v_{\mathrm{bf} t} \sin \phi \sin \theta+v_{\mathrm{bf} p} \cos \theta\right) \hat{\mathbf{y}} \\
& \left.+\left(v_{\mathrm{bf} n} \sin \phi+v_{\mathrm{bft}} \cos \phi\right) \hat{\mathbf{z}}\right) / v_{\mathrm{b} 0} .
\end{aligned}
$$

The Magnus force can similarly be expressed as

$$
\mathbf{F}_{\mathbf{M}}=1 / 2 \rho\left(\pi r^{2}\right) C_{L} v_{\mathrm{b}}^{2} \hat{\mathbf{s}}_{\mathrm{b}},
$$

where $C_{L}$ is the lift coefficient, and the unit vector $\hat{\mathbf{s}}_{\mathbf{b}}$ is given by

$$
\hat{\mathbf{s}}_{\mathbf{b}}=\hat{\omega}_{\mathbf{b}} \times \hat{\mathbf{v}}_{\mathbf{b}}
$$

The initial flight values of $\omega_{b}$ and $\omega_{\mathbf{b}}$ are given by

$$
\omega_{\mathrm{b} 0}=\omega_{\mathrm{bf}}=\left(\omega_{\mathrm{bf} t}^{2}+\omega_{\mathrm{bf} p}^{2}\right)^{1 / 2},
$$

and

$$
\begin{aligned}
\hat{\omega}_{\mathrm{b} 0}= & \left(\left(\omega_{\mathrm{bf} t} \cos \phi\right) \hat{\mathbf{x}}-\left(\omega_{\mathrm{bf} t} \sin \phi \cos \theta+\omega_{\mathrm{bf} p} \sin \theta\right) \hat{\mathbf{y}}\right. \\
& \left.+\left(\omega_{\mathrm{bf} p} \cos \theta-\omega_{\mathrm{bft}} \sin \phi \sin \theta\right) \hat{\mathbf{z}}\right) / \omega_{\mathrm{b} 0} .
\end{aligned}
$$

The drag and lift coefficients depend on the Reynolds number, the nature of the ball's surface, and in general on the ball's speed and spin. Experimental values obtained by Bearman and Harvey, ${ }^{12}$ for hexagonal dimpled British golf balls, were found to give good agreement with respect to measurements of the carry, which refers to the total distance the ball travels before landing, of driven golf balls. Therefore interpolated values of these coefficients were used in the analysis.

The linear acceleration, a, of the golf ball during its flight is given by Newton's second law:

$$
\mathbf{a}=\left(\mathbf{F}_{\mathbf{G}}+\mathbf{F}_{\mathbf{D}}+\mathbf{F}_{\mathbf{M}}\right) / m \text {. }
$$

In order to determine the change in the drag and the lift coefficients over the flight of the golf ball, the change in the spin of the golf ball is required. The magnitude of the angular acceleration, $\alpha$, for a golf ball has been determined in wind tunnel tests. These measurements show that the spin rate of a golf ball on landing is approximately $75 \%$ of its 
initial spin rate. Smits and Smith ${ }^{16}$ gave the following empirical expression for $\alpha$ :

$$
\alpha=-(0.00002)\left(\omega_{\mathrm{b}} v_{\mathrm{b}} / r\right),
$$

which was used in the analysis. The orientation of the spin will be taken to be fixed throughout the flight. Given the initial linear and angular velocities of the golf ball, the linear and angular accelerations during its flight, and the Bearman and Harvey ${ }^{12}$ lift and drag coefficients, the trajectory and carry of the golf ball can be determined.

After the ball hits the ground it will bounce several times and then ideally roll a certain distance before coming to rest. The total distance traveled after landing is referred to as the run. Penner, ${ }^{5}$ using results from Daish, ${ }^{3}$ modeled the run, $\Lambda$, of a golf ball for the case of central impacts and the subsequent two-dimensional trajectory and landing velocity. Applying this model to the general case of off-center impacts and the resulting three-dimensional trajectory and landing velocities results in the following;

$$
\boldsymbol{\Lambda}=\left\{(4 / g) v_{\text {brv }} v_{\text {brh }}+v_{\text {brh }}^{2} /\left(2 g \mu_{r}\right)\right\} \hat{\mathbf{h}},
$$

where $v_{\text {brv }}$ and $v_{\text {brh }}$ are the vertical and horizontal components of the rebound velocity (i.e. after the first bounce), $\mu_{r}$ is the coefficient of friction between the golf ball and the fairway, and $\hat{\mathbf{h}}$ is in the direction of the horizontal component of the landing velocity. This direction will be given by

$$
\hat{\mathbf{h}}=\left(v_{\mathrm{bq} x} \hat{\mathbf{x}}+v_{\mathrm{bqz}} \hat{\mathbf{z}}\right) / v_{\mathrm{bqh}},
$$

where $v_{\mathrm{bqx}}$ and $v_{\mathrm{bqz}}$ are the $x$ and $z$ components of the landing velocity, $\mathbf{v}_{\mathbf{b q}}$, and $v_{\text {bqh }}$ is given by

$$
v_{\mathrm{bqh}}=\left(v_{\mathrm{bqx}}^{2}+v_{\mathrm{bqz}}^{2}\right)^{1 / 2} \text {. }
$$

The vertical component of the rebound velocity can be expressed as

$$
v_{\text {brv }}=e_{f}\left|v_{\text {bqy }}\right|,
$$

where $v_{\text {bqy }}$ is the vertical component of the landing velocity and $e_{f}$, the coefficient of restitution between the golf ball and the fairway, was found by Penner ${ }^{5}$ to be approximated by the following empirical equation:

$$
e_{f}=0.510-0.0375\left|v_{\text {bqy }}\right|+0.000903\left|v_{\text {bqy }}\right|^{2} .
$$

The horizontal component of the rebound velocity, $v_{\text {bqh }}$, was shown by Daish to be given by

$$
v_{\mathrm{brh}}=\left(5 v_{\mathrm{bqh}}-2 r \omega_{\mathrm{bqh}}\right) / 7
$$

where $\omega_{\text {bqh }}$, the horizontal component of the spin that is perpendicular to the horizontal component of the landing velocity will be given by

$$
\omega_{\mathrm{bqh}}=\omega_{\mathrm{bq}} \cdot(\hat{\mathbf{h}} \times \hat{\mathbf{y}}) .
$$

Using this model for the run in the case of a two-dimensional trajectory and landing velocity, Penner found a reasonable fit with the experimental results of Williams ${ }^{17}$ for a value of $\mu_{r}$ equal to 1.0 and therefore this was the value used in the analysis. It is important, however, to state that this model for the run should only be treated as a rough approximation to the real behavior of a golf ball after landing.

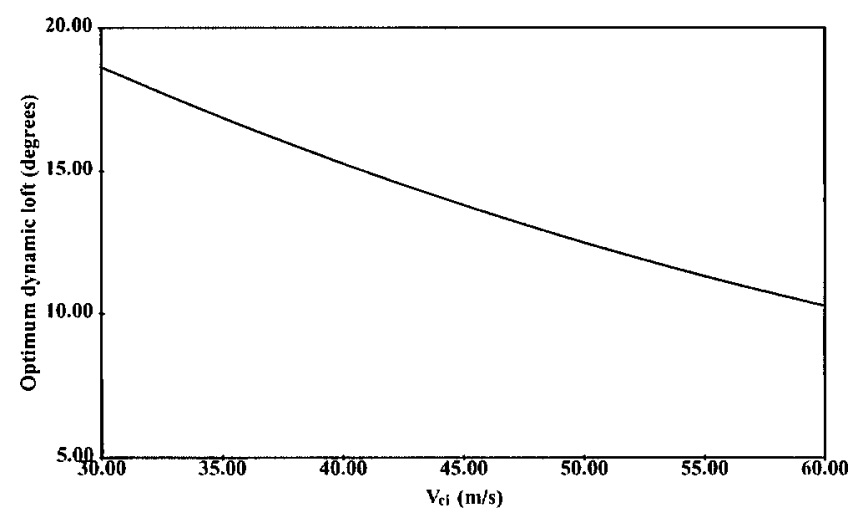

Fig. 5. The dependence of the optimum dynamic loft on impact speed, $v_{\text {ci }}$, for a clubhead of mass $200 \mathrm{~g}$ and a volume of $250 \mathrm{cc}$.

\section{RESULTS}

\section{A. Optimum loft of the clubhead}

Before considering off-center impacts and the curvature of the clubface, the loft to be used in the clubhead model needs to be determined. This was accomplished by determining the clubhead loft which will result in the maximum overall drive distance for impacts at the center of the face. Equations (19)-(23) were therefore solved for various loft angles, $\theta$, and initial clubhead speeds, $v_{\mathrm{ci}}$ for impacts with $\phi=0$. The resulting initial launch velocity components were then used along with Eqs. (29) - (45) to determine the carry and run of the launched golf ball. The trajectories were calculated numerically using a step size of $0.001 \mathrm{~s}$, which resulted in a calculation uncertainty of less than $0.1 \mathrm{yd}$ in the carry and drive of the golf ball. The distances in this paper will be given in yards as it is the standard unit of measurement in the game of golf. The clubhead lofts which resulted in the maximum drive distances for various initial clubhead speeds were thereby determined. It should be noted that the lofts calculated are correctly referred to as the dynamic lofts or the lofts of the clubface at impact. In general, due to the flex of the club shaft, the dynamic loft is several degrees greater than the clubface loft which is normally specified on the clubhead.

Figure 5 shows the results for the dependence of the optimum dynamic loft on the initial clubhead speed, $v_{\text {ci }}$, given for a clubhead mass of $200 \mathrm{~g}$ and a clubhead volume of 250 cc. As an example, for an initial clubhead speed of $45 \mathrm{~m} / \mathrm{s}$, the optimum dynamic loft is $13.3^{\circ}$ for the above clubhead parameters. These particular values for the clubhead mass, volume, dynamic loft, and impact speed will be referred to as the reference clubhead for the remainder of the paper. The overall drive distances for the optimum dynamic lofts for the various clubhead speeds are shown in Fig. 6 . For the $45-\mathrm{m} / \mathrm{s}$ reference clubhead speed, the drive distance is $233 \mathrm{yds}$. These results show that in general the optimum dynamic loft decreases with increasing clubhead speed, which corresponds with current golf practice. Analysis by Penner, ${ }^{5}$ where the clubhead was treated as a thin plate, gave values similar to those shown in Figs. 5 and 6. For example, for the same clubhead mass of $200 \mathrm{~g}$ and initial speed of $45 \mathrm{~m} / \mathrm{s}$ the optimum dynamic loft for the thin plate model was found to be $13.1^{\circ}$ and the corresponding drive distance was $232 \mathrm{yds}$. 


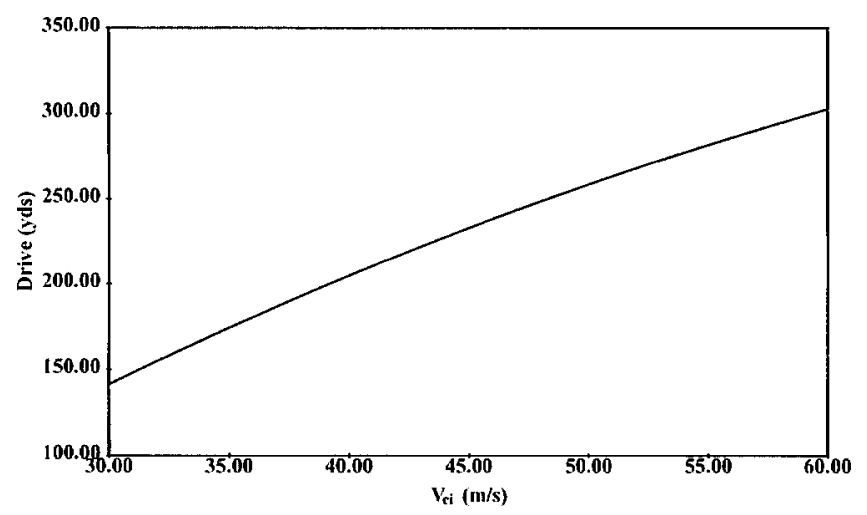

Fig. 6. The dependence of the drive distance on impact speed, $v_{\text {ci }}$, for a clubhead of mass $200 \mathrm{~g}$, volume of $250 \mathrm{cc}$, and with the optimum dynamic loft.

\section{B. Optimum radius of curvature}

In order to determine the amount of curvature that needs to be given to the clubface of the reference clubhead, so as to have off-center impacted golf balls ending up stopping in the middle of the fairway, the specific impact point needs to be selected. An impact point $2.0 \mathrm{~cm}$ from the center of the face was arbitrarily chosen and although the values which will be presented in this paper only apply to this specific impact point, the conclusions arrived at will hold in general.

As discussed in Sec. II there are two opposing effects which will determine the direction and magnitude of the tangential velocity component of the clubface at the impact point, $v_{a t}$. These were referred to as the angle and gear effects. The magnitude of each of these effects will depend on the parameters of the golf clubhead, including the curvature of the clubface, and on the position of the impact point. For example, consider the reference clubhead with the impact point $2.0 \mathrm{~cm}$ from the center of the face. Figure 7 shows how $v_{a t}$ varies with the radius of curvature of the clubface. As is shown, $v_{a t}$ is positive for radii of curvature greater than $14.1 \mathrm{~cm}$. A positive $v_{a t}$ corresponds to the gear effect dominating. For curvature of radii less than $14.1 \mathrm{~cm}, v_{a t}$ is negative and the angle effect will be dominant. The overhead view of the resulting golf ball trajectories and runs for the reference clubhead are shown for various radii of curvature in Fig. 8. As is shown for a radius of curvature of $14.1 \mathrm{~cm}$ the impacted golf ball will not have any sidespin and will fly

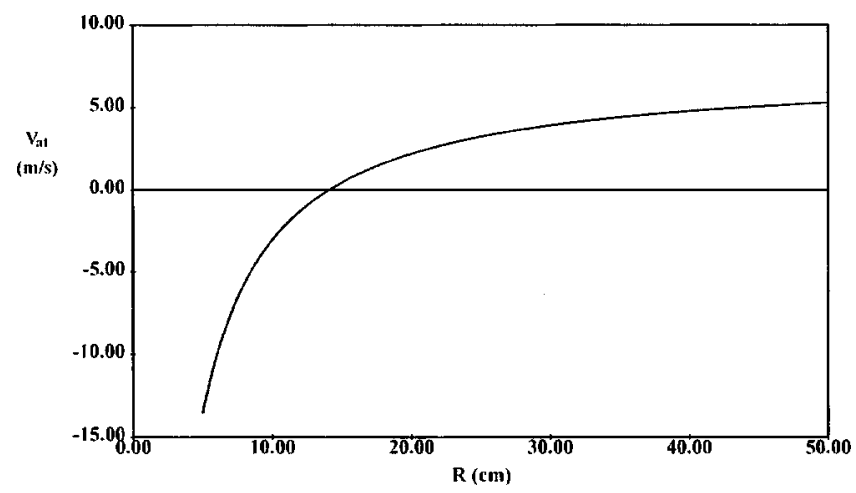

Fig. 7. The dependence of the tangential velocity component of the clubface, $v_{a t^{\prime}}$, on the radius of curvature of the clubface, $R$, for an impact point $2.0 \mathrm{~cm}$ from the clubface center.

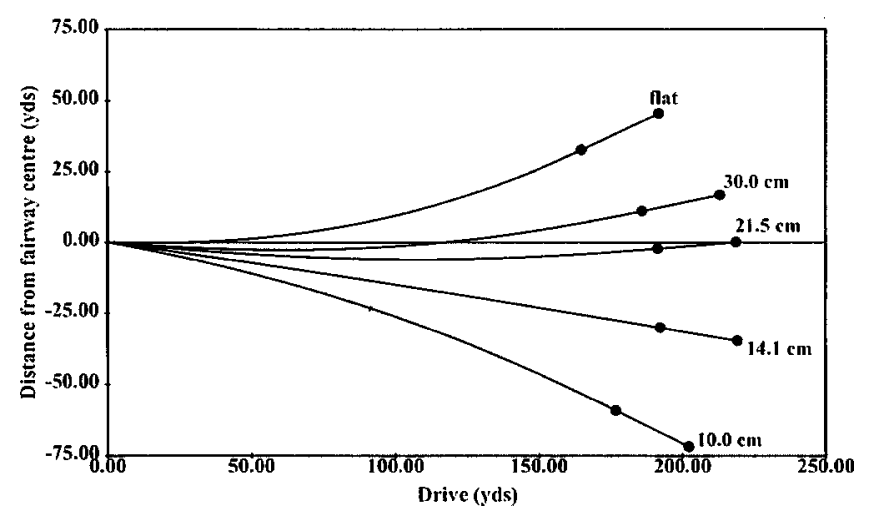

Fig. 8. Overhead view showing the dependence of the trajectory $(-)$ and run $(\bullet \bullet)$ of a golf ball, for various radii of curvature of the clubface, for an impact point $2.0 \mathrm{~cm}$ from the clubface center.

straight, normal to the clubface at the impact point. For radii of curvature less than $14.1 \mathrm{~cm}$, the ball will veer further away from the center of the fairway. For radii of curvature greater than $14.1 \mathrm{~cm}$, the golf ball will curve back toward the center of the fairway. The optimum radius of curvature for the reference clubhead model is found to be $21.5 \mathrm{~cm}$ for the given impact point, as the golf ball ends up coming to rest in the middle of the fairway. For radii of curvature greater than $21.5 \mathrm{~cm}$, the golf ball will curve back toward the center of the fairway but will overshoot and land off center. This value for the optimal radius of curvature which has been determined is in good agreement with current clubhead design practice. For example, Maltby ${ }^{2}$ used a mechanical golfer to determine empirically that the optimal radii of curvature for the clubfaces of drivers range from approximately $20.3 \mathrm{~cm}$ (8 in.) to $27.9 \mathrm{~cm}(11 \mathrm{in}$.).

\section{Dependence on clubhead parameters}

General conclusions with regards to the dependence of the optimum radius of curvature for the clubface on clubhead volume, mass, and initial speed can be reached with the given clubhead model. First, the volume of the reference clubhead was varied and the optimum radii of curvature for an impact point $2.0 \mathrm{~cm}$ from the center of the clubface was determined. The optimum radius of curvature for the given impact point was found to increase with clubhead volume. The result is shown in Fig. 9 with radii of curvature increas-

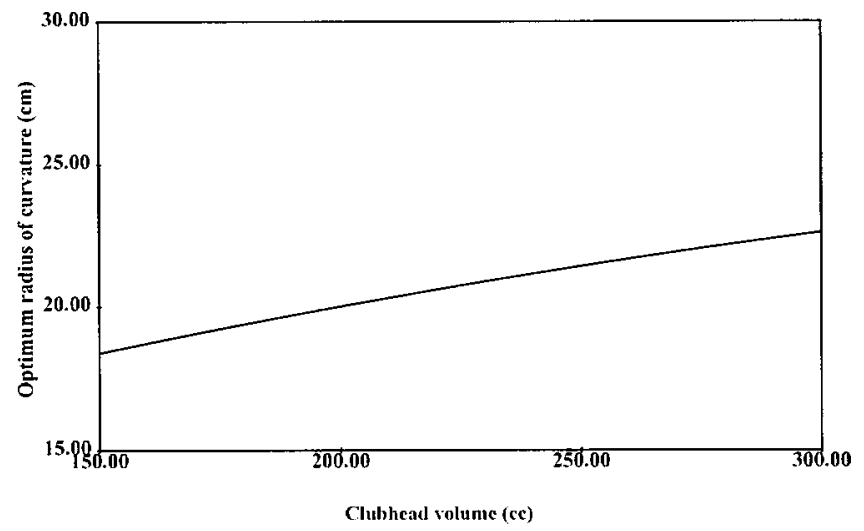

Fig. 9. The dependence of the optimum radius of curvature on clubhead volume for an impact point $2.0 \mathrm{~cm}$ from the clubface center. 


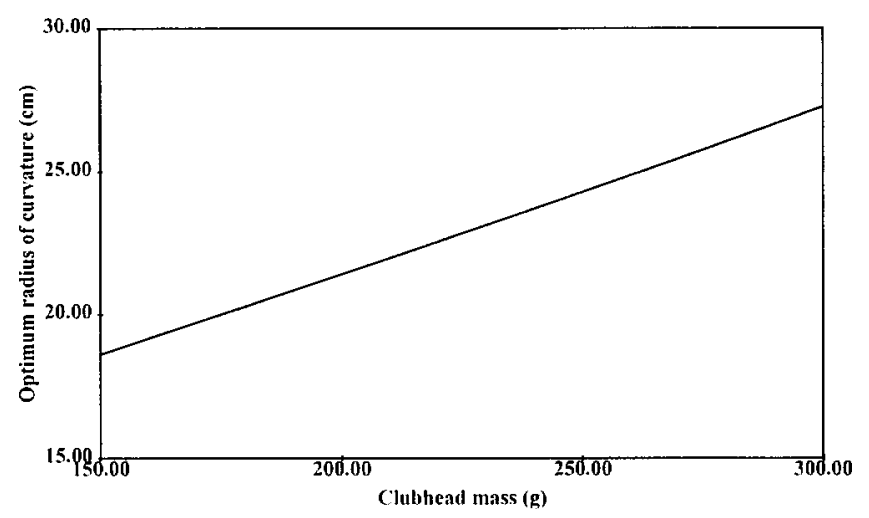

Fig. 10. The dependence of the optimum radius of curvature on clubhead mass for an impact point $2.0 \mathrm{~cm}$ from the clubface center.

ing from $18.4 \mathrm{~cm}$ for a clubhead volume of $150 \mathrm{cc}$ to 22.6 $\mathrm{cm}$ for a volume of $300 \mathrm{cc}$. In general it may be concluded that the radius of curvature of the clubface should increase with increasing clubhead volume. This follows from the fact that increasing the volume will increase the moment of inertia of the clubhead which, in general, will result in reducing the angular velocity imparted to the clubhead. This will lead to a reduced gear effect and will therefore result in a reduction of the sidespin imparted to the golf ball. Therefore less compensation for the gear effect is required leading to an increased radius of curvature of the clubface or in other terms a reduced clubface curvature.

The effect of increasing the mass of the reference clubhead model on the optimum radius of curvature was also determined. A result similar to that of increasing the volume was found. As shown in Fig. 10, optimum radii of curvature, for an impact point $2.0 \mathrm{~cm}$ from the center of the clubface, increased from $16.2 \mathrm{~cm}$ for a clubhead mass of $150 \mathrm{~g}$ to 23.0 $\mathrm{cm}$ for a clubhead mass of $300 \mathrm{~g}$. As with increasing the volume, increasing the mass results in an increased moment of inertia, a reduced gear effect, and therefore an increased optimum radius of curvature for the clubface.

Finally, the effect of initial clubhead speed on the optimum radius of curvature, for an impact point $2.0 \mathrm{~cm}$ from the center of the clubface, was determined. The result is shown in Fig. 11 with the optimum radius of curvature decreasing with increasing clubhead speed, with values dropping from $29.5 \mathrm{~cm}$ for a clubhead speed of $30 \mathrm{~m} / \mathrm{s}$ to $19.6 \mathrm{~cm}$ at $60 \mathrm{~m} / \mathrm{s}$. It would be expected in general that increasing the

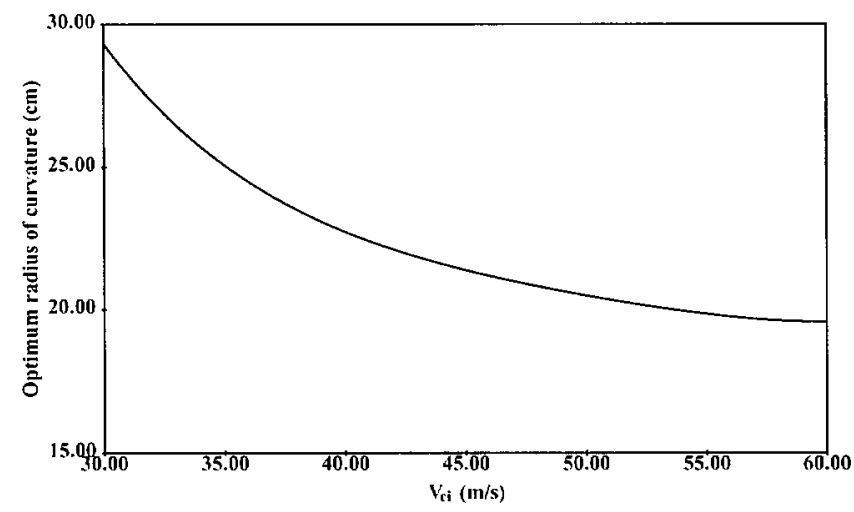

Fig. 11. The dependence of the optimum radius of curvature on impact speed, $v_{\text {ci }}$, for an impact point $2.0 \mathrm{~cm}$ from the clubface center.

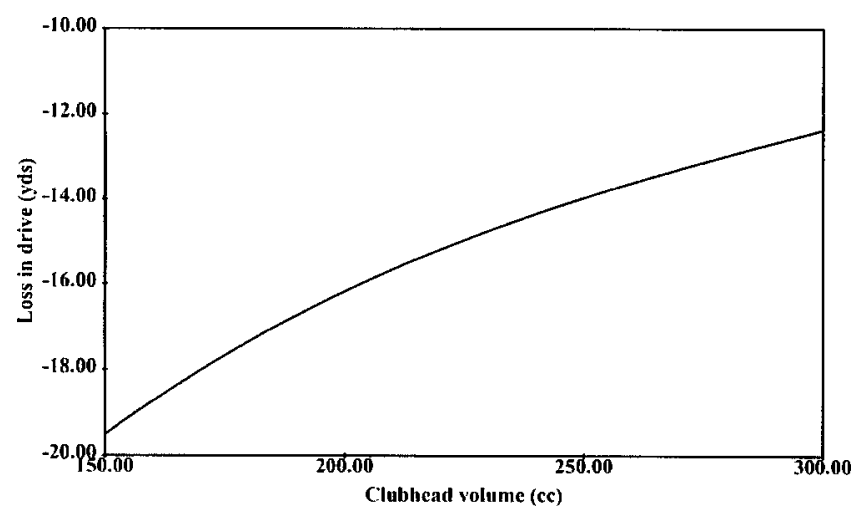

Fig. 12. The dependence of the loss in drive distance on clubhead volume for an impact point $2.0 \mathrm{~cm}$ from the clubface center.

clubhead speed would result in requiring a smaller radius of curvature for the clubface. This follows from the fact that the magnitude of the gear effect is directly proportional to the impact speed of the clubhead. Therefore, with greater sidespin applied to the ball, the clubface would need to be curved more, or the radius of curvature would need to be less, in order to compensate.

Given that the curvature of the clubface can be optimized for a given clubhead volume and mass so as to cause an off-center impacted golf ball to end up in the middle of the fairway, the question arises as to the benefit of a larger clubhead. To partially answer this question, the loss in the drive distance needs to be considered. For example, for impacts at the center of the clubface the drive distance will be independent of the volume of the clubhead. However, this is not the case for off-center impacts. Figure 12 shows the loss in the drive distance for the reference clubhead for impact points $2.0 \mathrm{~cm}$ from the clubface center in the case where the clubface curvature has been optimized. As shown for a clubhead of volume $150 \mathrm{cc}$, the net loss in the drive distance is 19.5 yds while for a clubhead volume of $300 \mathrm{cc}$ it is only 12.4 yds. This dependence is due to the optimum radius of curvature of the clubface increasing with increasing volume, resulting in the golf ball flying straighter for larger volume clubheads.

\section{CONCLUSION}

The impact between the clubhead of a driver and a golf ball was modeled and the effect of the convex clubface was determined. It was found that by taking into account the sidespin imparted to the golf ball and the resulting Magnus effect, the curved clubface will compensate for the gear effect for off-center hits. For the reference clubhead model, with a typical clubhead impact speed of $45 \mathrm{~m} / \mathrm{s}$, the optimum radius of curvature of the clubface was found to be approximately $21.5 \mathrm{~cm}$ for an impact point $2.0 \mathrm{~cm}$ from the center of the clubface. It was found that increasing the clubhead mass or volume results in a reduced gear effect and therefore required an increase in the radius of curvature for the clubface. It was also found that increasing the impact speed of the clubhead resulted in an increased gear effect and therefore required a decrease in the radius of curvature of the clubface.

The clubface model used in the analysis allows the clubface to be optimized for only one off-center impact point, namely $2.0 \mathrm{~cm}$ in the case of the above results. In general, the optimum clubface shape would not be cylindrical and 
more than a single impact point would need to be considered. In addition, the change in the orientation of the clubhead during impact would be expected to lead to greater optimal radii of curvature. However the general dependence of the optimum clubface curvature on clubhead volume, mass, and impact speed presented in this paper would be expected to hold.

${ }^{1}$ A. Cochran and J. Stobbs, The Search for the Perfect Swing (Lippincott, New York, 1968).

${ }^{2}$ R. Maltby, Golf Club Design, Fitting, Alterations and Repair (Maltby Newerk, 1990).

${ }^{3}$ C. B. Daish, The Physics of Ball Games (English U.P., London, 1972).

${ }^{4}$ T. P. Jorgensen, The Physics of Golf (Springer-Verlag, New York, 1994).

${ }^{5}$ A. R. Penner, "The physics of golf: The optimum loft of a driver," Am. J. Phys. 69 (5), 563-568 (2001).

${ }^{6}$ T. Olsavsky, "The effects of driver head size on performance," Proceedings of the 1994 World Scientific Congress of Golf, edited by A. J. Cochran and M. R. Farrally (E \& FN Spon, London, 1994).

${ }^{7}$ M. J. Sullivan and T. Melvin, "The relationship between golf ball construction and performance," in Ref. 6.
${ }^{8} \mathrm{~W}$. Goldsmith, Impact The Theory and Physical Behaviour of Colliding Solids (Arnold, London, 1960).

${ }^{9}$ R. M. Brach, "Rigid Body Collisions," J. Appl. Mech. 56, 133-138 (1989).

${ }^{10}$ P. C. Chou, D. Liang, J. Yang, and W. Gobosh, "Contact forces, coefficient of restitution, and spin rate of golf ball impact," in Ref. 6 .

${ }^{11}$ B. B. Lieberman and S. H. Johnson, "An analytical model for ball-barrier impact," in Ref. 6.

${ }^{12}$ P. W. Bearman and J. K. Harvey, "Golf Ball Aerodynamics," Aeronaut. Q. 27, 112-122 (1976).

${ }^{13} \mathrm{H}$. Erlichson, "Maximum projectile range with drag and lift, with particular application to golf," Am. J. Phys. 51 (4), 357-361 (1983).

${ }^{14}$ W. M. MacDonald and S. Hanzely, "The physics of the drive in golf," Am. J. Phys. 59 (3), 213-218 (1991).

${ }^{15}$ J. J. McPhee and G. C. Andrews, "Effect of sidespin and wind on projectile trajectory with particular application to golf," Am. J. Phys. 56 (10), 933-939 (1988).

${ }^{16}$ A. J. Smits and D. R. Smith, "A new aerodynamic model of a golf ball in flight," in Ref. 6.

${ }^{17} \mathrm{D}$. Williams, "Drag force on a golf ball in flight and its practical significance," Q. J. Mech. Appl. Math. XII (3), 387-392 (1959). 